\title{
Sawtooth-like oscillations of black hole accretion disks
}

\author{
Ryoji Matsumoto $^{1}$ and Mami Machida ${ }^{2}$ \\ ${ }^{1}$ Department of Physics, Faculty of Science, Chiba University 1-33, Yayoi-cho, Inage-ku, \\ Chiba, 263-8522 Japan \\ ${ }^{2}$ Division of Theoretical Astronomy, National Astronomical Observatory of Japan, 2-21-1, \\ Osawa, Mitaka, Tokyo 181-8588, Japan \\ email: matumoto@astro.s.chiba-u.ac.jp, mami@th.nao.ac.jp
}

\begin{abstract}
We studied the origin of quasi-periodic oscillations (QPOs) of X-rays in black hole candidates by three-dimensional global resistive magnetohydrodynamic simulations of accretion disks. Initial state is a rotating disk threaded by weak toroidal magnetic fields. General relativistic effects are simulated by using the pseudo-Newtonian potential. When the temperature of the outer disk decreases, the accreting matter accumulates into an inner torus.

We found that the inner torus is deformed into a crescent shape and that it shows sawtoothlike oscillations of magnetic energy with frequency $3-5 \mathrm{~Hz}$ when the mass of the black hole is $10 M_{\odot}$. The magnetic energy inside the torus is amplified until magnetic reconnection suddenly releases the accumulated magnetic energy. A new cycle of the oscillation starts when magnetic energy is amplified again. We found that high frequency QPOs with frequency around $100 \mathrm{~Hz}$ in stellar mass black holes are excited when sawtooth-like oscillation appears in the inner torus.
\end{abstract}

Keywords. accretion, accretion disks - MHD - QPO

\section{Introduction}

Quasi-periodic oscillations (QPOs) are sometimes observed in black hole candidates when the source is in low/hard state or during the transition from low/hard state to high/soft state. The frequency of QPOs in low/hard state $(1-10 \mathrm{~Hz}$ in stellar mass black holes) increases as the source luminosity increases. Figure 1 schematically shows the evolution of accretion disks in low/hard state and during the hard-to-soft state transition. Solid curves show thermal equilibrium curves of accretion disks obtained by Abramowicz et al. (1995). When the accretion rate exceeds $0.1 \dot{M}_{E}$, where $\dot{M}_{E}$ is the Eddington accretion rate, optically thin solution disappears.

Machida et al. (2006) showed by three-dimensional global resistive magnetohydrodynamic (MHD) simulations that the outer region of the disk where radiative cooling exceeds the heating shrinks in the vertical direction. The disk remains optically thin because magnetic pressure supports the disk. High frequency QPOs (HFQPOs) with frequency $\sim 100 \mathrm{~Hz}$ appear in such X-ray hard, luminous disks. HFQPOs accompany low frequency QPOs (LFQPOs). In this paper we report the results of global three-dimensional resistive MHD simulations of black hole accretion disks during the hard-to-soft state transition.

\section{Numerical methods}

We numerically solved resistive MHD equations in cylindrical coordinates $(\varpi, \varphi, z)$ by using a modified Lax-Wendroff method with artificial viscosity. We assumed anomalous resistivity $\eta=\eta_{0}\left[\max \left(v_{d} / v_{c}-1,0\right)\right]^{2}$ where $v_{d}=j / \rho$ is the electron-ion drift speed 
Accretion
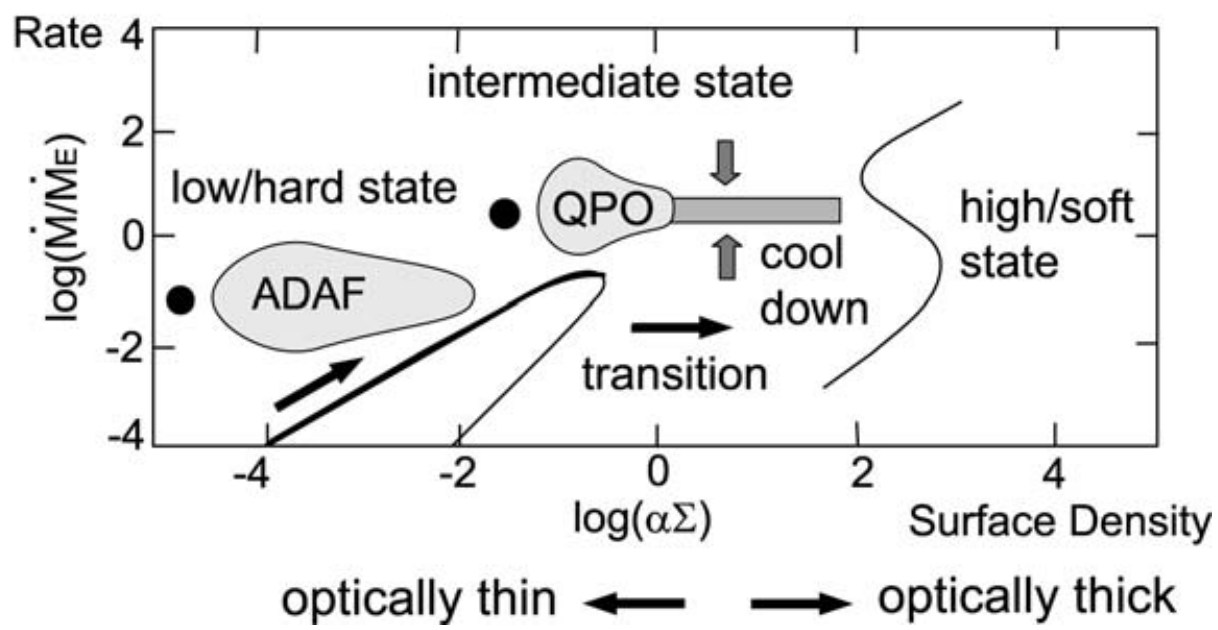

Figure 1. A schematic picture showing the hard-to-soft state transition. Solid curves denote thermal equilibrium curves of accretion disks in $\Sigma-\dot{M}$ plane, where $\Sigma$ is surface density and $\dot{M}$ is the accretion rate. When mass accretion rate exceeds the critical value for the onset of the cooling instability, outer regions of the disk shrinks in the vertical direction due to cooling.

and $v_{c}$ is the threshold above which the anomalous resistivity sets in. Here $j$ and $\rho$ are current density and matter density, respectively. Radiative cooling is neglected. General relativistic effects are simulated by using the pseudo-Newtonian potential $\phi=-G M /(r-$ $r_{s}$ ) where $M$ is the mass of the black hole, $r=\left(\varpi^{2}+z^{2}\right)^{1 / 2}$, and $r_{s}$ is the Schwarzschild radius.

The initial state is a single temperature, polytropic disk with polytropic index $n=3 / 2$ and the angular momentum $L \propto \varpi^{0.46}$ threaded by weak toroidal magnetic fields. The initial strength of magnetic field is parametrized by the ratio of gas pressure to magnetic pressure $\beta_{0}=P_{\text {gas }} / P_{\text {mag }}$ at the initial pressure maximum of the disk at $(\varpi, z)=\left(\varpi_{0}, 0\right)$. We take $\varpi_{0}=35 r_{s}$ and $\beta_{0}=100$. The initial temperature of the disk at the pressure maximum is $T_{0}=10^{9} \mathrm{~K}$. This temperature is an order of magnitude smaller than that of our previous simulations (Machida \& Matsumoto 2003). The initial disk is embedded in a low density, spherical, hot isothermal halo.

The magnetic Reynolds number $R_{\mathrm{m}}=c r_{s} / \eta_{0}$ and the threshold for the anomalous resistivity are taken to be $R_{m}=2000$ and $v_{c}=0.9 c$, respectively. In the following, the unit of length is $r_{\mathrm{s}}$, and the unit of time is $t_{0}=r_{\mathrm{s}} / c=10^{-4} M_{10}$ s where $M_{10}=$ $M /\left(10 M_{\odot}\right)$.

The number of grid points is 250 in radial direction, 32 in azimuthal direction and 384 in vertical direction. The grid size is $\Delta \varpi=\Delta z=0.1$ when $0<\varpi, z<10$, and otherwise increases with $\varpi$ and $z$. We imposed absorbing boundary condition at $r=2 r_{s}$. Other boundaries are free boundary where waves can be transmitted.

\section{Numerical results}

Figure 2 shows the isosurface of density in the inner region $(\varpi<25)$ at $t=58000 t_{0}$. As the disk infalls due to the angular momentum transport by MRI (magnetorotational instability) driven turbulent magnetic field, an inner torus is formed around $\varpi=8 r_{s}$. We found that the inner torus is deformed into a crescent shape. 


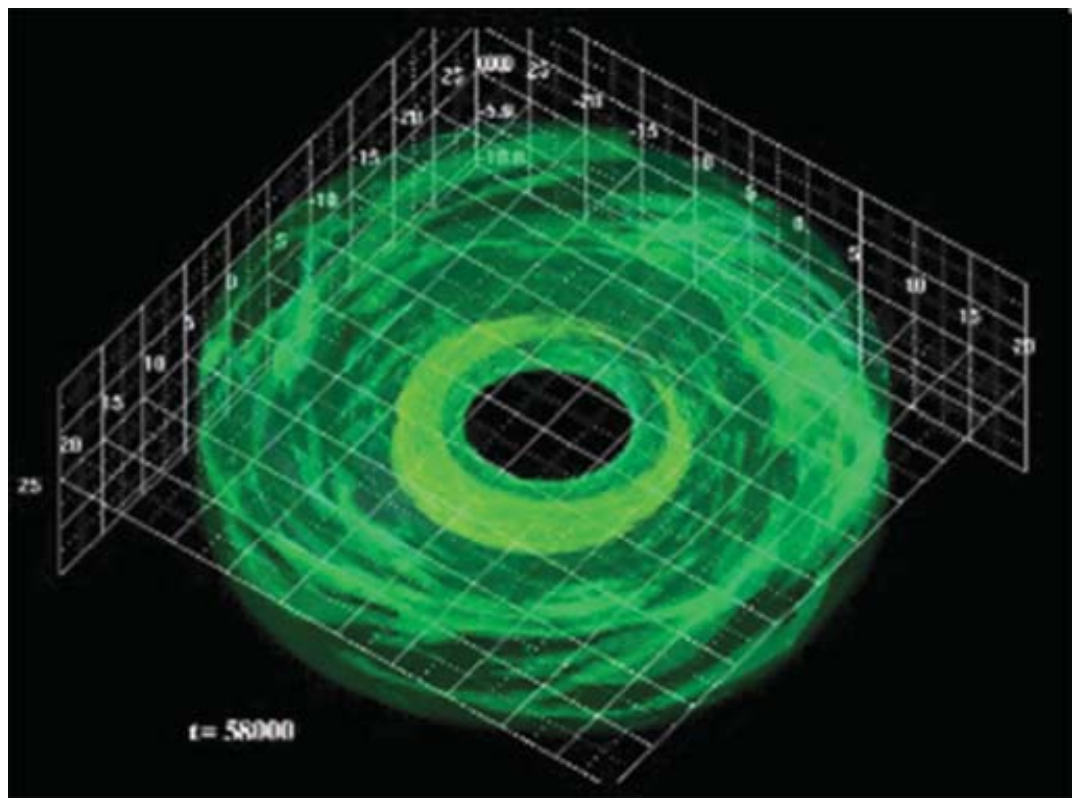

Figure 2. Isosurface of density at $t=58000 t_{0}$.

Figure 3 shows the time evolution of the magnetic energy (solid curves) and the Joule heating rate (dashed curves) integrated in the inner torus. They show a sawtooth-like oscillation with period $\sim 2500 \mathrm{rs} / \mathrm{c}=0.25 M_{10} \mathrm{~s}$. The Joule heating rate increases when the magnetic energy decreases. It indicates that magnetic energy is dissipated. Sawtooth like oscillations are excited in nonlinear systems when an instability and dissipation coexists. When the dissipation is large, the system approaches to a quasi-steady state. On the other hand, when the dissipation is small, the energy accumulated by the growth of the instability is suddenly released by catastrophic events such as magnetic reconnection. When the energy release brings the system back to the original state, the accumulation and release of the energy creates a sawtooth-like oscillation. Sawtooth oscillations are observed in Tokamak fusion reactors, in which plasma is disrupted by magnetic reconnection.

In the inner torus formed in our simulation, magnetic energy is amplified when onearmed ( $m=1$ where $m$ is the azimuthal mode number), non-axisymmetric density distribution appears. Since the dense blob in a differentially rotating disk stretches the magnetic field lines in azimuthal direction and deform them into a bisymmetric spiral shape, current sheets are formed inside the spiral channel (Machida \& Matsumoto 2003). When the current density exceeds the critical density for the onset of the anomalous resistivity, magnetic reconnection taking place in the current sheet releases the accumulated magnetic energy. Subsequently, the $m=1$ mode and the magnetic energy grows again.

Figure 4 shows the power density spectrum (PDS) of the time variation of the mass accretion rate measured at $\varpi=2.5 r_{s}$ when $M=10 M_{\odot}$. A broad peak around $5 \mathrm{~Hz}$ corresponds to the sawtooth-like oscillation. We found that when the large-amplitude sawtooth oscillation appears, two high frequency QPOs with frequency ratio 2:3 appear around $100 \mathrm{~Hz}$. The large amplitude sawtooth-like oscillation excites the high frequency oscillations of the inner torus. 


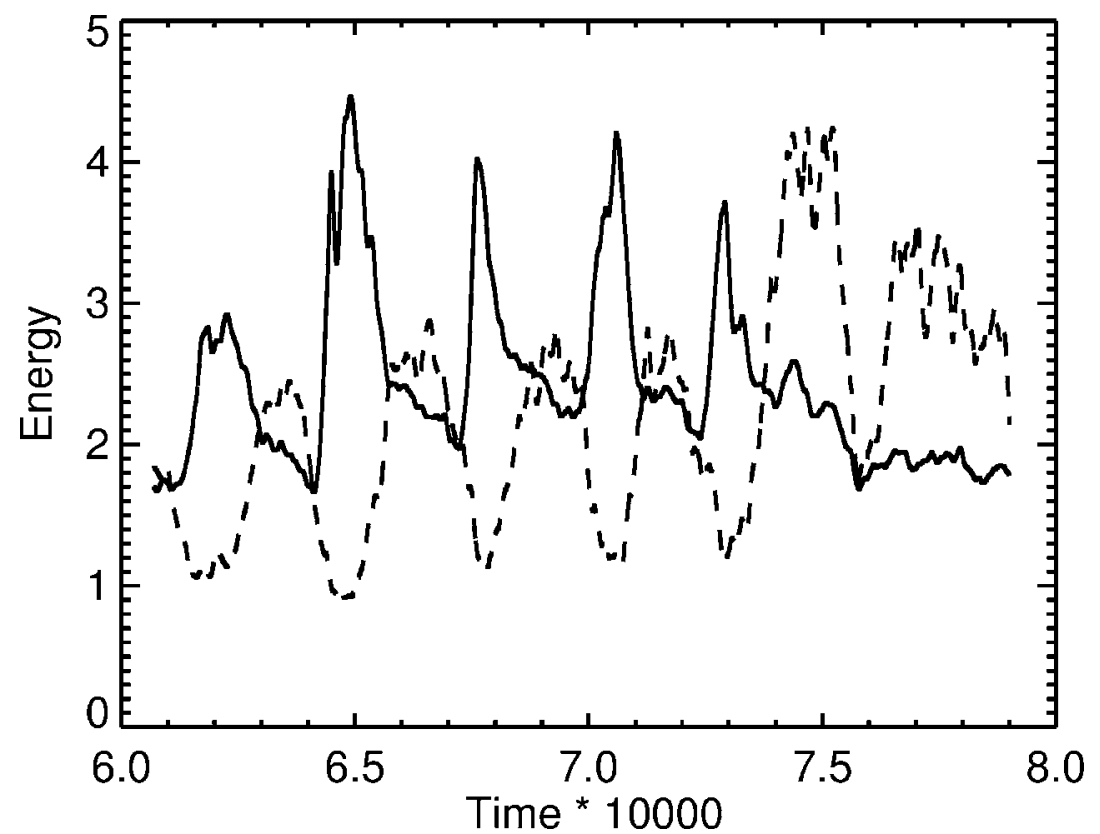

Figure 3. Time evolution of the magnetic energy (solid curve) and the Joule heating rate (dashed curve).

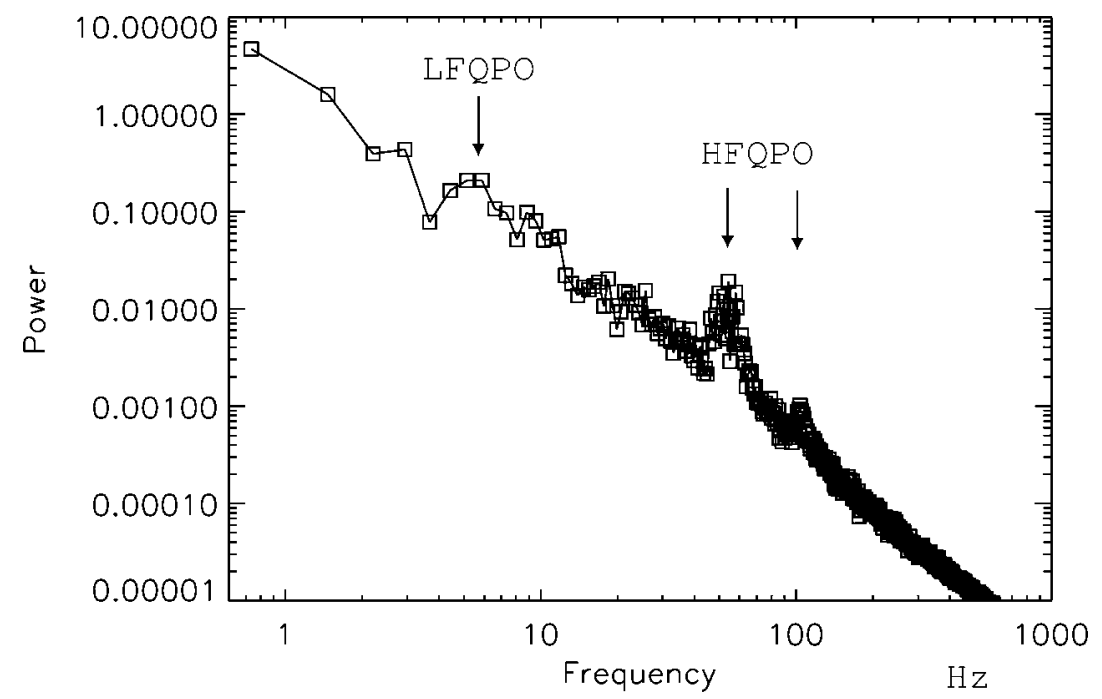

Figure 4. Power density spectrum of the time variation of the mass accretion rate at $\varpi=2.5 r_{s}$ when the mass of the black hole is $10 M_{\odot}$.

\section{Summary and discussion}

We showed that when the temperature of the outer disk in black hole accretion flows decreases due to cooling, sawtooth-like oscillation is excited in the inner torus formed inside $10 r_{s}$. The frequency of the sawtooth oscillation is $3-5 \mathrm{~Hz}$ and comparable to that of LFQPOs observed in black hole candidates. The period of the sawtooth oscillation is determined by the growth time of magnetic fields, typically 10 rotation period of the inner torus. During the growth of magnetic fields, the inner torus is deformed into a crescent 
shape. When the magnetic energy becomes comparable to the thermal energy, magnetic energy accumulated in the inner torus is released by magnetic reconnection. The sawtooth oscillation excites high frequency oscillations of the inner torus with frequency around $100 \mathrm{~Hz}$. Our numerical results are consistent with that HFQPOs are observed during the hard-to-soft state transitions in black hole candidates and that they accompany LFQPOs.

\section{Acknowledgements}

Numerical computations were performed by using VPP5000 at National Astronomical Observatory, Japan. This work is supported in part by the Grants-in-Aid for Scientific Research of the Ministry of Education, Culture, Sports, Science and Technology (RM: 17030003) and JSPS Fellowships for young scientists (MM: 17-1907, 18-1907).

\section{References}

Abramowicz, M. A., Chen, X., Kato, S., Lasota, J.-P., Regev, O. 1995, ApJ, 438, L37

Machida, M., Matsumoto, R. 2003, ApJ, 585, 429

Machida, M., Nakamura, K. E., Matsumoto, R. 2006, PASJ, 58, 193

Elisabete de Gouveia Dal Pino: Can the sawtooth instability feed a dynamo action in the disc, so that a poloidal magnetic field component can be amplified from the large scale toroidal component and then raise from the disc into the corona?

Ryoji Matsumoto: Magnetorotational instability drives the dynamo. During the growing state of the sawtooth oscillation magnetic fields are amplified up to $\beta \sim 1$, where $\beta$ is the ratio of gas pressure to magnetic pressure. Since our simulation includes vertical gravity, magnetic fields buoyantly escape from the disc and generate poloidal magnetic fields. Although we solve the full magnetohydrodynamic equations instead of reduced dynamo equations, the amplification and maintenance of magnetic fields in discs are similar to those in classical dynamo theory.

FUKUN LIU: I would like to ask two questions. 1. What's the dependence of QPOs on the radial extent of torus? 2. Can you find multiple QPOs with harmonic relationship e.g. 2 : 3, firstly discovered by Marek Abramowicz and collaborators, and in AGNs by our recent work (see poster S238-129)?

Ryoj Matsumoto: 1 . We did the simulations with different radial extent of torus of $20 R_{\mathrm{g}}$ and $10 R_{\mathrm{g}}$. The frequency is independent of the radial size of torus. 2 . Yes, we identified two QPOs with $2: 3$ frequency ratio in power-spectral density.

Alexander Zakharov: Did you try to fit the jet formation with your approach?

Ryoji Matsumoto: Our simulations produce funnel-wall outflows and magnetically driven disc winds. The speed of the disc wind is typically $\sim 0.05 c$. 


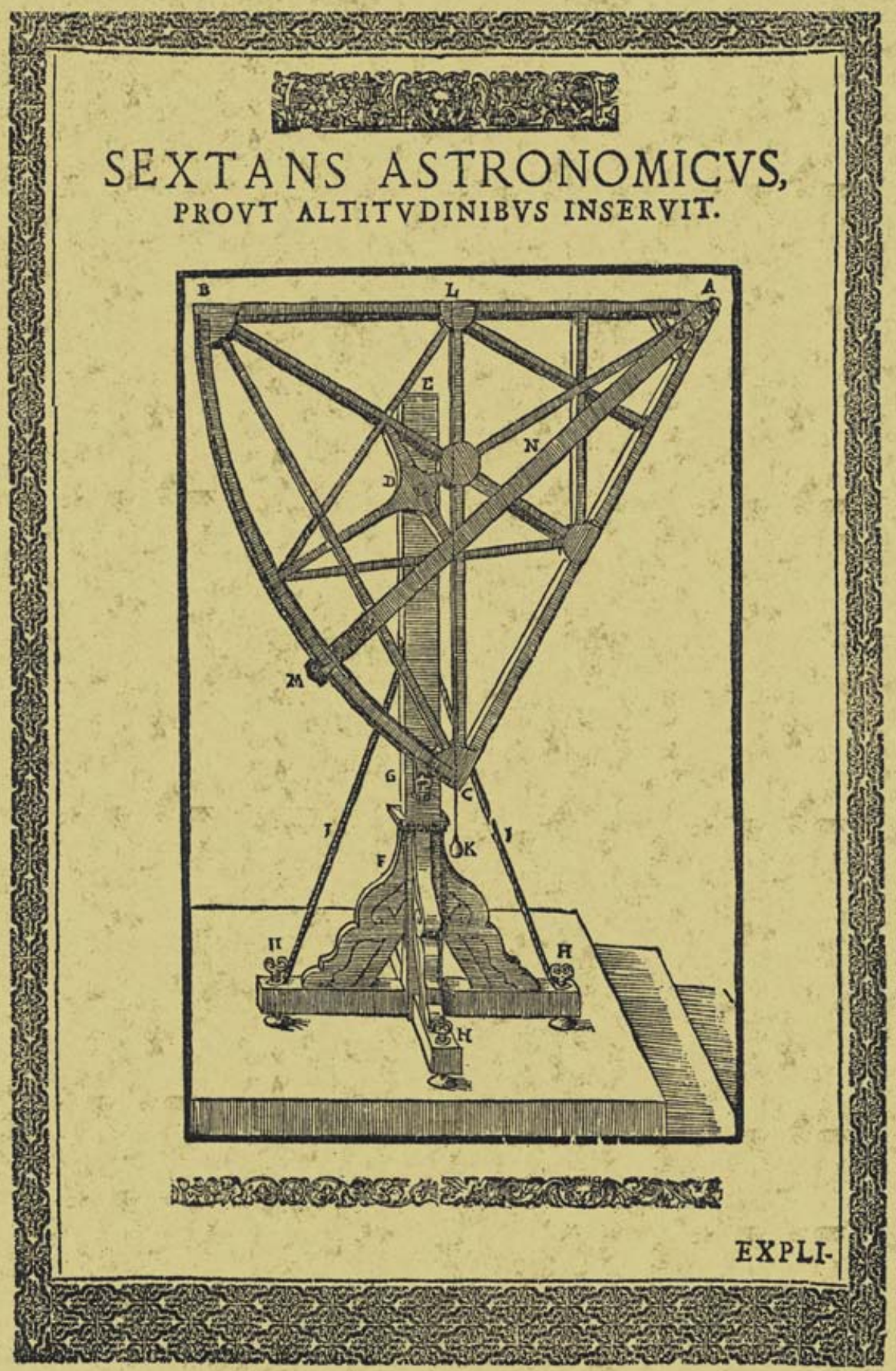

